\title{
Magnetic Properties of Multilayer Thin Film with Ising-Like Ordering
}

\author{
E. BîRSAN* \\ Department of Physics, "Lucian Blaga" University, Dr. Ioan Ratiu Str. No. 5-7, 550012 Sibiu, Romania
}

(Received October 28, 2009; in final form January 15, 2010)

\begin{abstract}
In the last years, the study of the antiferromagnetic-ferromagnetic interfaces was a very exciting topic in the thin film area. Using the Monte Carlo technique (by applying the standard Metropolis algorithm) and the extended Heisenberg model with low dipolar interaction, the influence of the antiferromagnetic base layer on the magnetic properties of the multilayer thin film is investigated, in the ferromagnetic region of the phase diagram of the system. For each ferromagnetic layer individually, we study the physical quantities of interest (assuming particular physical conditions): the out-of-plane magnetization, the magnetic susceptibility and the specific heat and we point out the Ising-like ordering of the magnetic spins. The obtained numerical results show that the critical temperature of the individual layers decreases as we approach the base antiferromagnetic layer and consequently, the stability of the ferromagnetic phase increases with the distance from the base layer.
\end{abstract}

PACS numbers: 75.10.Hk, 75.70.Ak, 75.40.Mg

\section{Introduction}

During the last decades the magnetic materials have become a research domain of special interest, mainly due to their applications in technology. Consequently, the study of magnetic thin films registered an important development, from both theoretical and experimental point of view [1-5]. In this context, many scientific studies (theoretical and experimental) have been dedicated to the multilayered magnetic structures [6-8], considering the interesting interface phenomena that can appear in this kind of systems. At the same time, the numerical approaches in this domain attract a special attention $[9,10]$, representing a good instrument to test the compatibility of the new proposed physical models and to predict the new potential phenomenon that can appear in certain physical conditions.

The aim of this paper is to investigate the magnetic properties of a multilayer ferromagnetic (FM) thin film characterized by an AFM-FM interface (the magnetic spins of the base layer are described by an antiferromagnetic (AFM) ordering), using the Monte Carlo method as a numerical method to study the magnetic behavior of the physical system presented below.

The paper is structured as follows: in Sect. 2 we present the physical model we assume, Sect. 3 is dedicated to the numerical algorithm that stands at the base of the numerical simulation, in Sect. 4 we present the nu-

* e-mail: birsan.eugen@gmail.com merical results obtained in our study, and in Sect. 5 the final conclusions are formulated.

\section{The physical model}

As we mentioned above we deal with AFM-FM interface composed of a number of ferromagnetic layers over an antiferromagnetic one. This type of interface systems have been extensively studied in the last years [11-13] due to their important applications in the magnetic data storage industry and in the magnetoresistive devices technology. In thin films, having regard to the systems dimensions, it is essential to take into account the anisotropical phenomena that can occur. Consequently, in these conditions, numerous studies have been devoted to various anisotropic models which were developed, or to different experimental observations that emphasize the anisotropic character of some phenomena that appear in this kind of systems $[5,11,14]$.

Our study consists of some numerical simulations on a layered structure characterized by the AFM-FM interface. The physical model is described by the following extended Heisenberg Hamiltonian:

$$
\begin{aligned}
H & =-\sum_{\langle i, j\rangle} J_{\alpha_{i} \beta_{j}} \boldsymbol{S}_{i} \cdot \boldsymbol{S}_{j}-\sum_{\langle i, j\rangle} A_{\alpha_{i} \beta_{j}} S_{i z} S_{j z} \\
& +\sum_{i, j} D_{\alpha_{i} \beta_{j}} T_{i j} .
\end{aligned}
$$

The first term of the above Hamiltonian defines the direct exchange interaction between the adjacent spins of the lattice and is responsible for the magnetism of the 
system. Here $J_{\alpha i \beta j}>0$ is the corresponding exchange constant and $\boldsymbol{S}_{i}$ represents the spin vector that characterizes the magnetic spin at the site $i$ of the lattice. The second term of the Hamiltonian of the system describes the exchange anisotropy interaction between the neighboring spins $\left(A_{\alpha i \beta j}>0\right.$ represents the parameter of anisotropic exchange interaction and $S_{i z}$ is the $z$ component of the spin vector) and behaves as an easy-axis anisotropy interaction that favors the alignment of the magnetic spins along the $z$ axis. The last term of the Hamiltonian means the long range dipolar interaction, leading to shape anisotropy [15], where $T_{i j}$ is given by the expression

$$
T_{i j}=\frac{\boldsymbol{S}_{i} \cdot \boldsymbol{S}_{j}}{r_{i j}^{3}}-3 \frac{\left(\boldsymbol{S}_{i} \cdot \boldsymbol{r}_{i j}\right)\left(\boldsymbol{S}_{j} \cdot \boldsymbol{r}_{i j}\right)}{r_{i j}^{5}} .
$$

Here, $D_{\alpha i \beta j}>0$ represents the dipolar interaction parameter and $r_{i j}$ is the distance between the spins involved in the interaction. In contrast with the first two terms, where the interactions act between the nearest neighbor spins of the system, in the dipolar term we take into account all the pairs of spins of the lattice. The last two terms of the Hamiltonian (1) manifest competitive behaviors: the exchange anisotropy favors, as we already have mentioned, the $z$ orientation of the spins, whereas the dipolar interaction tends to align the spin in the plane $x O y$ and determines the magnetic ordered cluster forming. On the other hand, given the dominant $z$ orientation of the spins, induced by the exchange anisotropy, the dipolar fields tend to anti-align the magnetic spins [16]. This behavior overlaps with the ferromagnetic nature of the local Heisenberg interactions and determines the magnetic features of the system. Consequently, the three terms of the above presented Hamiltonian, act in a competitive manner in our system (taking into account the antiferromagnetic ordering of the base layer). Considering that we stand in the ferromagnetic region of the phase diagram of the system, the last two terms of the Hamiltonian lead to the variation of the ferromagnetic phase stability in the upper layers. In the end of this section, it is also important to note that the physical model parameters are indexed in accordance to the layer to which belong the spins involved in that interaction.

\section{The numerical algorithm}

In this paper we use the Monte Carlo technique [17], by applying the Metropolis algorithm, in order to study the antiferromagnetic base layer influence on the upper layers magnetic behavior. We apply the numerical algorithm to cubic magnetic spins structure characterized by the lattice constant $a=1$. The lattice consists of $N_{z}=4$ layers, indexed with numbers from 0 to 3 , the base layer (indexed by 0 ) having a fixed antiferromagnetic ordering. Each layer contains $N_{x} \times N_{y}=40 \times 40$ magnetic spins, with the modulus $S=1$, randomly oriented around a fixed point. Our cubic lattice of magnetic spins is characterized by open boundary conditions (OBC) in the $z$ direction and periodic boundary conditions $(\mathrm{PBC})$ in the $x$ and $y$ directions. We run $10^{3}$ steps per spin for the thermalization of the system and other $10^{3}$ steps per spin to evaluate the physical quantities of interest: the out-of-plane magnetization, the out-of-plane magnetic susceptibility and the specific heat, individually calculated for each layer. The $z$ component of the magnetization per spin is

$$
\tilde{m}_{z}=\frac{\sum_{i=1}^{N} S_{i z}}{N} .
$$

Here $S_{i z}$ are the $z$ components of the unitary magnetic spins vectors and $N=N_{x} N_{y}$ represents the number of spins in each layer. The normalized out-of-plane magnetization per spin is calculated by the expression

$$
m_{z}=\frac{\tilde{m}_{z}}{\tilde{m}_{z, \text { sat }}},
$$

where $\tilde{m}_{z \text {,sat }}$ means the magnetization per spin at saturation. The out-of-plane magnetic susceptibility and the specific heat are given by the following relations:

$$
\begin{aligned}
& \chi_{z}=N \frac{\left(\left\langle\tilde{m}_{z}^{2}\right\rangle-\left\langle\tilde{m}_{z}\right\rangle^{2}\right)}{k_{\mathrm{B}} T}, \\
& c=N \frac{\left\langle E^{2}\right\rangle-\langle E\rangle^{2}}{k_{\mathrm{B}} T^{2}},
\end{aligned}
$$

where $T$ is the absolute temperature of the system (measured in $\left.J S^{2} / k_{\mathrm{B}}\right), k_{\mathrm{B}}$ is the Boltzmann constant, considered unitary and $E$ is the energy per spin (measured in $J S^{2}$ ), given by the relation

$$
\langle E\rangle=\frac{\langle H\rangle}{N} .
$$

As we have already specified, the main purpose of this study is to find out the influence of the base layer (characterized by the antiferromagnetic ordering of the spins) on the magnetic properties of the upper layers treated individually. Therefore we study the individual magnetic behavior of the superior layers, in the ferromagnetic region of the phase diagram (qualitatively presented in Fig. 1). This region is characterized by the Ising-like ordering of the magnetic spins (orientated perpendicular to the film plane). The red segment (the first from the left) corresponds to the ferromagnetism-paramagnetism phase transition which occurs in the system with increasing temperature. We expect that the critical temperatures of the superior layers are different, that confirm the different influence of the antiferromagnetic base layer on the upper layers magnetic ordering. This is the reason to determine for each layer individually the physical quantities of interest.

\section{The simulation results}

In our previous paper [18] we have focused our attention on the behavior of the above presented system in the mixed phase region of the phase diagram. In contrast to this, in the present study we want to investigate the properties of the multilayer thin film in the ferromagnetic region of the phase diagram, where the spins 


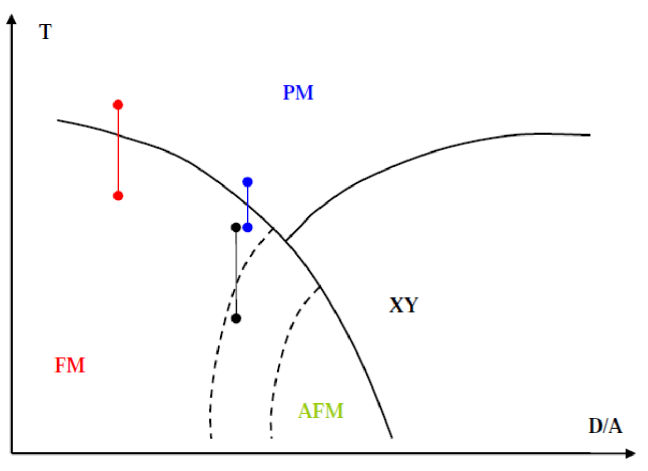

Fig. 1. The phase diagram corresponding to the investigated system (qualitative aspect).

have an out-of-plane magnetic ordering, that represents a specific feature of ultrathin films at low temperatures [19-21]. In this region of the phase diagram, the influence of the base antiferromagnetic layer is weaker than in the mixed phase zone and the ferromagnetic nature of the magnetic ordering of the upper layers are preserved.

In this section we present the numerical simulation results obtained for different values of the physical parameters. We have in view two different situations: in the first case we consider a constant parameter of the exchange anisotropy, and in the second case we assume the existence of an ascending gradient of the exchange anisotropy, if we approach the free surface of the film.

\subsection{Uniform exchange anisotropy}

In the thin films area, the anisotropic character of the physical interactions which appear in the studied systems is crucial. Thus, various studies have been dedicated to investigate the anisotropic behavior of these systems as a function of different factors (temperature or film thickness, for example), achieving significant results [22], which highlighted the different magnetic behavior of thin films, compared with the corresponding bulk systems. Moreover, in these conditions, to elucidate the specific phenomena which appear in these systems, it is necessary to determine the magnetic arrangement near the interfaces of different materials (for example: two different FM layers, a magnetic and a nonmagnetic material, or an FM-AFM interface) [23].

The numerical simulations offer the advantage of being able to study, using the same physical model, different dominant characteristics of a system by appropriate choice of parameters involved in the model used. As we mentioned above, our principal goal is to study the influence of the AFM base layer on the upper FM layers, individually. In this case, we assume for our numerical study, the following set of the physical parameters (setting symbolized with $\Theta$ ): $J_{00}=-J=-1$, $J_{11}=J_{22}=J_{33}=J=0.8, J_{01}=1, J_{12}=J_{23}=$ $J^{\prime}=0.75, A_{11}=A_{22}=A_{33}=A=1.1, A_{01}=A_{12}=$ $A_{23}=A^{\prime}=1.1, D_{11}=D_{22}=D_{33}=D=0.12$,
$D_{01}=D_{12}=D_{23}=D^{\prime}=0.12$. In these physical conditions we study the magnetic behavior of the superior layers $(1,2,3)$ by calculating the out-of-plane magnetization (Fig. 2), the out-of-plane magnetic susceptibility (Fig. 3) and the specific heat (Fig. 4) - versus temperature, for each layer individually, except for the base layer, characterized by a fixed antiferromagnetic arrangement. In Fig. 2 we observe the saturated character of the out-of-plane magnetization at low temperatures $(T<1)$ and its tendency to cancel at high temperatures $(T>1.8)$, which suggests an Ising-like orientation of the spins at low temperatures, magnetic ordering that breaks with increasing temperature (the system moving to a disordered phase).

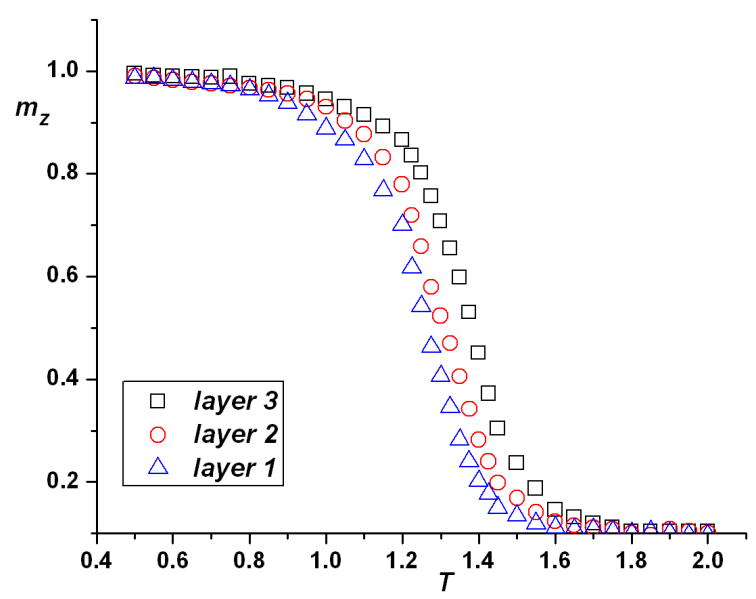

Fig. 2. The out-of-plane magnetization versus temperature for the $\Theta$ setting of the physical parameters.

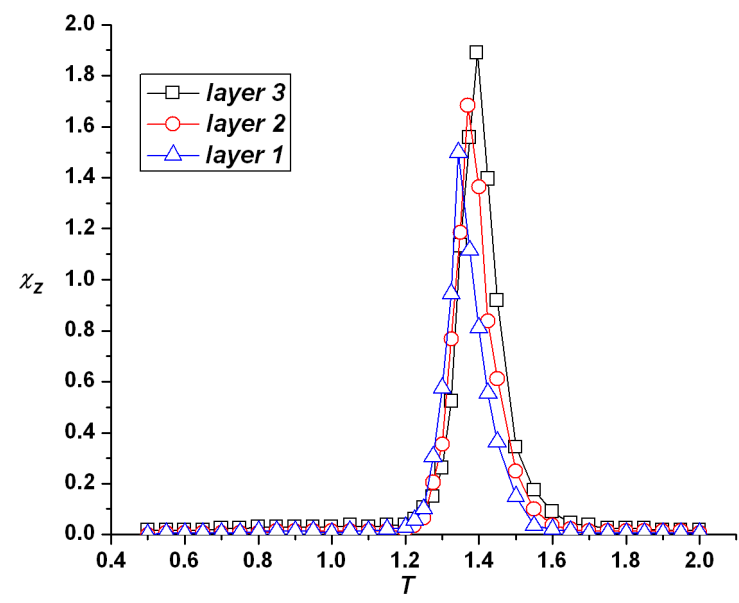

Fig. 3. The out-of-plane magnetic susceptibility versus temperature for the $\Theta$ setting of the physical parameters.

The critical temperature, specific to this magnetic phase transition can be determined from the local critical point of the graph that represents the out-of-plane magnetic susceptibility versus temperature (Fig. 3), or 


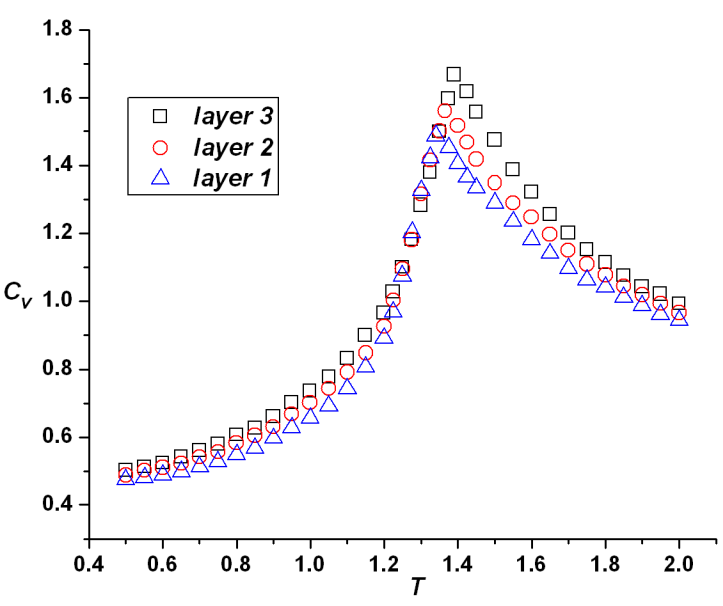

Fig. 4. Specific heat versus temperature for the $\Theta$ setting of the physical parameters.

from the position of the local critical point characteristic to the specific heat versus temperature, for each layer (Fig. 4). We can observe the critical temperature increase with the distance from the base layer (characterized by an antiferromagnetic ordering of the spins), as a proof of its decreasing influence. Therefore, in the upper layers (characterized by ferromagnetic ordering) the stability of the ordered phase increases with the distance from the base layer, so that the highest stability is observed for the third layer from the AFM-FM interface of the film.

In other words, we can remark that the highest instability of the ferromagnetic arrangement of the spins is obtained for the first ferromagnetic layer (localized at the interface between the base antiferromagnetic layer and the ferromagnetic domain of the thin film), where the influence of the base layer is maximal. In Table I we present, in a comparative manner, the critical temperatures obtained by interpreting the graphs of out-of-plane magnetic susceptibility and the specific heat versus temperature, respectively, where we find a better correlation of the critical temperature values, obtained for each layer in these two ways.

\section{TABLE I}

The critical temperature corresponding to the out-of-plane magnetic susceptibility maximum points and respectively to the specific heat, for the three layers in the case of $\Theta$ setting of the physical parameters.

\begin{tabular}{c|c|c}
\hline \hline Layer & $T_{\mathrm{C}}\left(\chi_{z}\right)$ & $T_{\mathrm{C}}\left(C_{V}\right)$ \\
\hline 3 & 1.3951 & 1.3902 \\
2 & 1.3699 & 1.3650 \\
1 & 1.3450 & 1.3401
\end{tabular}

This behavior seems to be quite natural, since the influence of the interface anisotropy decreases with the FM layer thickness. Thus, in systems like $\mathrm{CoO} /$ permal- loy multilayers, there was found a linear dependence of the exchange field with the inverse permalloy $(\mathrm{Py})$ layer thickness, which shows that the induced uniaxial anisotropy is of the nature of the interface anisotropy [11]. The short-range character of FM-AFM interactions which we consider in our model, is confirmed by the experimental results obtained for $[\mathrm{Pt} / \mathrm{Co}]-\mathrm{IrMn}$ multilayers [13]. Thus, it was observed that the insertion of a Pt spacer between the FM multilayers and the AFM layer leads to the appearance of a peak in the variation of the exchange field as a function of FM layer thickness, instead of the inversely proportional relationship mentioned above.

\subsection{Non-uniform exchange anisotropy}

In the real thin films, and particularly in the multilayered systems, the anisotropic character of some magnetic properties can be influenced by different factors: the roughness, the defects, the different nature of the magnetic layers $[22,23]$. Using the numerical approaches, it is possible to consider the aspects that involve the variations of the anisotropy that can occur for various reasons, by an appropriate setting of the physical parameters of the model used in our simulation.

Consequently, in this section, we extend our analysis, taking into account the variation of the anisotropic parameters in the system. Thus, in this case we assume the existence of non-uniform exchange anisotropy, increasing with the distance from the base layer. We repeat, using a similar method, the study presented in the previous subsection (4.1) in order to find for these physical conditions the magnetic behavior of the superior layers $(1,2,3)$. Thus, in this paragraph we use the following setting of the physical parameters, symbolized with $\Phi$ : $J_{00}=-J=-1, J_{11}=J_{22}=J_{33}=J=0.8, J_{01}=1$, $J_{12}=J_{23}=J^{\prime}=0.75, A_{11}=A=1.1, A_{22}=1.2$, $A_{33}=1.3, A_{01}=1.1, A_{12}=1.2, A_{23}=1.3, D_{11}=$ $D_{22}=D_{33}=D=0.12, D_{01}=D_{12}=D_{23}=D^{\prime}=0.12$.

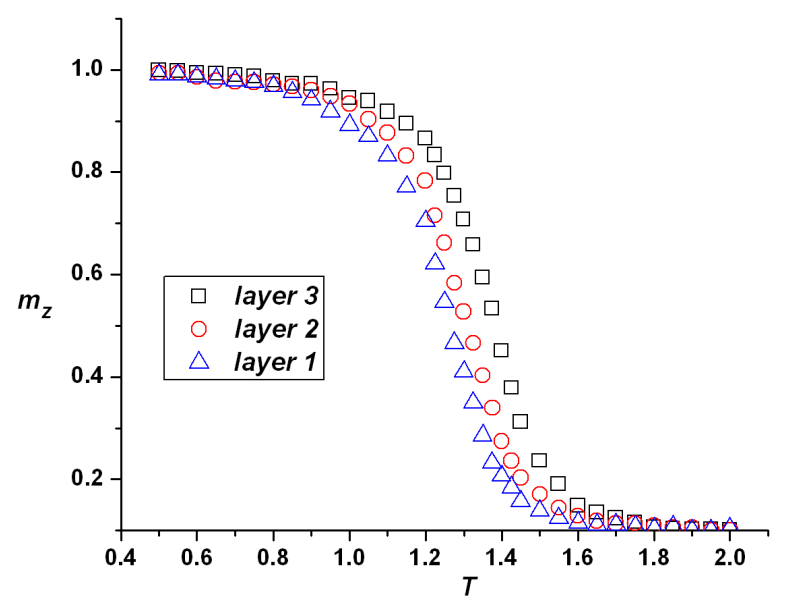

Fig. 5. Out-of-plane magnetization versus temperature for the $\Phi$ setting of the parameters. 


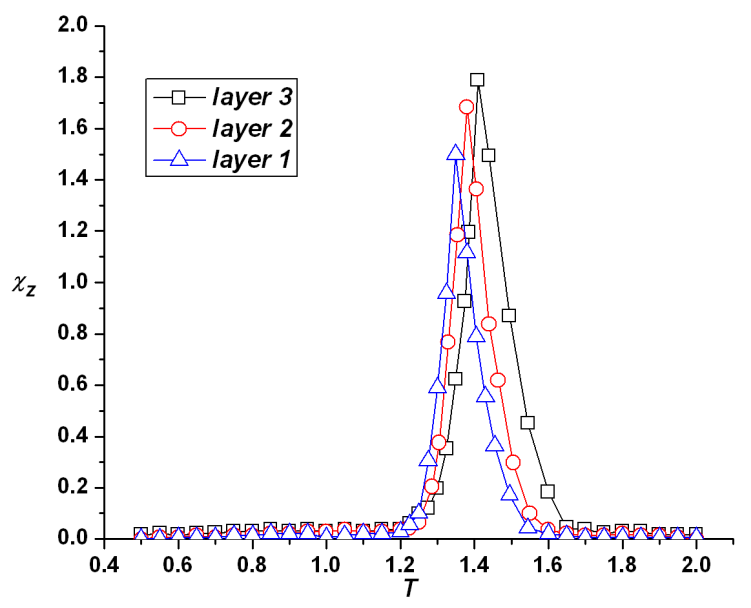

Fig. 6. Out-of-plane magnetic susceptibility versus temperature, for the $\Phi$ setting of the physical parameters.

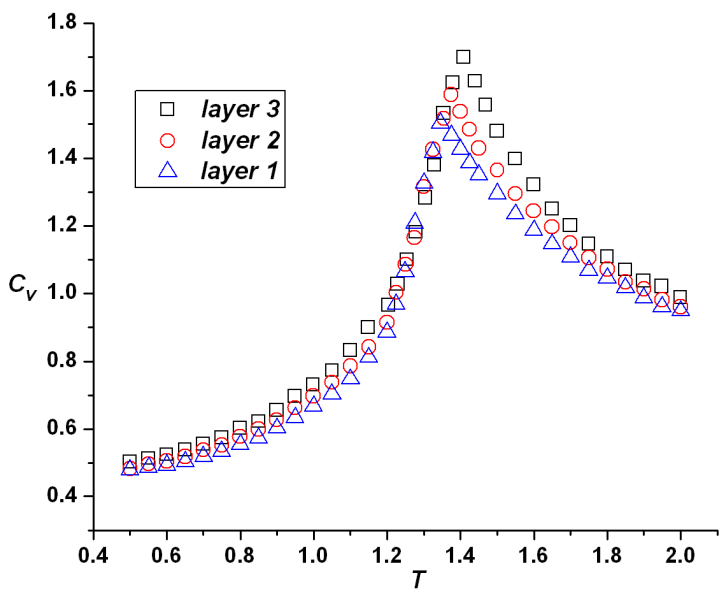

Fig. 7. Specific heat versus temperature, for the $\Phi$ setting of the physical parameters.

In Fig. 5, we present the out-of-plane magnetization versus temperature, which has the same form as in the previous case (saturated values at low temperatures and quasi-null values at high temperatures, in the thermal spectrum $T=[0.5 ; 2.0]$ ), with the difference that in this case the critical temperature (approximated by the inflexion point of the $m_{z}(T)$ graph) is somewhat higher, for all the three layers.

In Fig. 6 there is presented the magnetic susceptibility perpendicular to the film plane versus temperature, for the three upper layers involved in our study. It is observed here, in comparison with the previous case (where the exchange anisotropy was uniform), the increase of the critical temperatures for all the three layers, keeping the same behavior regarding the increasing stability of ferromagnetic phase with the distance from the base layer. These characteristics are confirmed also by the graph of the specific heat as a function of temperature (Fig. 7).
In Table II we present in a quantitative manner, the critical temperatures obtained by interpreting the graphs for the out-of-plane magnetic susceptibility and the specific heat versus temperature, respectively. We observe a better correlation of the critical temperature values, obtained in these two ways, for each layer.

\section{TABLE II}

The critical temperature corresponding to the out-of-plane magnetic susceptibility and respectively specific heat points of maximum, for the three layers, with $\Phi$ setting of the physical parameters table.

\begin{tabular}{c|c|c}
\hline \hline Layer & $T_{\mathrm{C}}\left(\chi_{z}\right)$ & $T_{\mathrm{C}}\left(C_{V}\right)$ \\
\hline 3 & 1.4102 & 1.4098 \\
2 & 1.3798 & 1.3749 \\
1 & 1.3499 & 1.3452
\end{tabular}

We can conclude for this subsection (4.2) that the non-uniform character of the exchange anisotropy leads to the enhancement of the critical temperatures that characterized the magnetic phase transitions, and consequently, the stability of the ferromagnetic phase in the upper layers is improved. Thus, in this region of the phase diagram, the multilayered nature of the system favors the out-of-plane orientation of the magnetization. In other words, these physical conditions lead to a cooperative behavior regarding the anisotropic and the direct exchange interactions, respectively.

\section{Conclusions}

Using the Monte Carlo technique, the base antiferromagnetic layer influence the upper layers of the Heisenberg magnetic thin films in the Ising-like ordering region of the phase diagram is investigated. We studied the magnetization, the magnetic susceptibility and the specific heat variations with temperature. We observed the critical temperature increase with the distance from the base layer. Therefore, in the ferromagnetic region of the phase diagram, the stability of the ferromagnetic state increases with the distance from the base layer, the most stable layer being the third layer from the AFM-FM interface of the film. This behavior is more acute if we assume non-uniform exchange anisotropy dependence in the layered film. Thus, in these physical conditions, the multilayered nature of the thin film favors the stability of the ferromagnetic ordering in the upper layers.

\section{References}

[1] G. Binasch, P. Grünberg, F. Saurenbach, W. Zinn, Phys. Rev. B 39, 4828 (1989).

[2] R. Allenspach, J. Magn. Magn. Mater. 129, 160 (1994).

[3] M. Kiwi, J. Magn. Magn. Mater. 234, 584 (2001). 
[4] J. Nogués, I.K. Schuller, J. Magn. Magn. Mater. 192 , 203 (1999).

[5] C.A.F. Vaz, J.A.C. Bland, G. Lauhoff, Rep. Prog. Phys. 71, 056501 (2008).

[6] P. Grünberg, Phys. Today, 31 (May 2001).

[7] F. Nolting, A. Scholl, J. Stöhr, J.W. Seo, J. Fompeyrine, H. Siegwart, J.-P. Locquet, S. Anders, J. Lüning, E.E. Fullerton, M.F. Toney, M.R. Scheinfein, H.A. Padmore, Nature 405, 767 (2000).

[8] O. Hellwig, A. Berger, J.B. Kortright, E.E. Fullerton, J. Magn. Magn. Mater. 319, 13 (2007).

[9] M.B. Taylor, B.L. Gyorffy, J. Phys., Condens. Matter 5, 4527 (1993).

[10] E.Y. Vedmedenko, H.P. Oepen, A. Ghazali, J.-C. S. Lévy, J. Kirschner, Phys. Rev. Lett. 84, 5884 (2000).

[11] S.M. Zhou, L. Sun, P.C. Searson, C.L. Chien, Phys. Rev. B 69, 024408 (2004).

[12] P.J. Jensen, H. Dreyssé, Phys. Rev. B 66, 220407(R) (2002).

[13] J. Sort, V. Baltz, F. Garcia, B. Rodmacq, B. Dieny, Phys. Rev. B 71, 054411 (2005).
[14] J. Moritz, S. van Dijken, J.M.D. Coey, Eur. Phys. J. B 45, 191 (2005).

[15] J. Kienert, S. Schwieger, K. Lenz, J. Lindner, K. Baberschke, W. Nolting, J. Magn. Magn. Mater. 316, e86 (2007).

[16] J.M. Deutsch, T. Mai, Phys. Rev. E 72, 016115 (2005).

[17] D.P. Landau, K. Binder, A Guide to Monte Carlo Simulations in Statistical Physics, Cambridge University Press, Cambridge 2000.

[18] E. Birsan, A. Dobrita, R. Chis, Mod. Phys. Lett. B 23, 643 (2009)

[19] D.P. Pappas, K.-P. Kämper, H. Hopster, Phys. Rev. Lett. 64, 3179 (1990).

[20] N.C. Koon, B.T. Jonker, F.A. Volkening, J.J. Krebs, G.A. Prinz, Phys. Rev. Lett. 59, 2463 (1987).

[21] R. Allenspach, A. Bischof, Phys. Rev. Lett. 69, 3385 (1992).

[22] M.T. Johnson, P.J.H. Bloemen, F.J.A. den Broder, J.J. deVries, Rep. Prog. Phys. 59, 1409 (1996).

[23] P.J. Jensen, K.H. Bennemann, Surf. Sci. Rep. 61, 129 (2006). 